\title{
Acknowledgement to Reviewers of Journal of Risk and Financial Management in 2019
}

JRFM Editorial Office

MDPI, St. Alban-Anlage 66, 4052 Basel, Switzerland; jrfm@mdpi.com

Received: 13 January 2020; Accepted: 13 January 2020; Published: 13 January 2020

The editorial team greatly appreciates the reviewers who have dedicated their considerable time and expertise to the journal's rigorous editorial process over the past 12 months, regardless of whether the papers are finally published or not. In 2019, a total of 183 papers were published in the journal, with a median time to first decision of 16 days and a median time from submission to publication of 41 days. The editors would like to express their sincere gratitude to the following reviewers for their generous contribution in 2019:

\author{
Abad-Segura, Emilio \\ Abidin, Sazali \\ Abudy, Menachem \\ Agliardi, Elettra \\ Agnieszka, Głodowska \\ Aguilar, Jean-Philippe \\ Aguilar-Fernández, María E. \\ Ahmad, Fayyaz \\ Akhtaruzzaman, Md \\ Alziyadat, Naser \\ Amaral, Teodosio Perez \\ Andraz, Jorge \\ Angelini, Eliana \\ Ante, Lennart \\ Anton, Sorin \\ Antulov-Fantulin, Nino \\ Antzoulatos, Angelos \\ Arbi, Lukman \\ Arraiano, Irene \\ Ashraf, Badar Nadeem \\ Atukeren, Erdal \\ Audrino, Francesco \\ Ausín Olivera, Maria Concepción \\ Avi-Yonah, Reuven \\ Baek, Seungho \\ Bahmani, Sahar \\ Bakhach, Amer \\ Bao, Jack \\ Bărbuță-Mişu, Nicoleta \\ Bariviera, Aurelio Fernandez \\ Barth, James R. \\ Barthélemy, Jean \\ Basov, Suren
}

\author{
Batrancea, Ioan \\ Bektic, Demir \\ Berger, Wolfram \\ Bernet, Beat \\ Błach, Joanna \\ Blue, Levon \\ Bögenhold, Dieter \\ Bolotov, Ilya \\ Boratyńska, Katarzyna \\ Borochkin, Alexander \\ Bostan, Ionel \\ Bosupeng, Mpho \\ Boubaker, Sabri \\ Bouri, Elie \\ Brooks, Robert \\ Brzozowski, Jan \\ Busu, Cristian \\ Butkus, Mindaugas \\ Cai, Jun \\ Cakici, Nusret \\ Calin, Adrian Cantemir \\ Čámská, Dagmar \\ Cansado-Bravo, Pablo \\ Caputo, Fabio \\ Caraballo, M. Angeles \\ Caraiani, Petre \\ Carini, Cristian \\ Cervelló-Royo, Roberto \\ Challet, Damien \\ Chan, Stephen \\ Chan, Leo H. \\ Chan, Leung Lung \\ Chang, Chia-Lin
}


Chang, Seong Yeon

Cheang, Gerald H. L.

Chen, Chun-Da

Chen, James M.

Chen, Qihui

Chen, Yi-Chang

Cheng, Mingmian

Chiang, Thomas

Chiappini, Helen

Choi, Seung Uk

Choongo, Progress

Ciarreta, Aitor

Cieslik, Ewa

Clemente, Gian Paolo

Clichici, Dorina

Coelho, Denis A.

Coelho, Luís Miguel Serra

Cristian, Paun

Croci, Ettore

Cumming, Douglas

Dabija, Dan-Cristian

Dang, Chongyu

De Pace, Pierangelo

Del Valle Soto, Carolina

Delcea, Camelia

Di Giacomo, Marina

Dobrovolskienė, Nomeda

Donadelli, Michael

Dorling, Danny

Drobyazko, Svetlana

Ehling, Paul

Ejdys, Joanna

Ekanayake, E. M.

Ellington, Michael

Ender, Manuela

Falk, Martin

Fanelli, Viviana

Farina, Vincenzo

Favilukis, Jack

Feinstein, Zachary

Feng, Runhuan

Ferreira, Paulo

Fillat, José L.

Fiori, Anna Maria

Floreani, Josanco

Foggin, Marc

Forero-Laverde, Germán

Forest, James

Fritzsche, Kerstin

Fusari, Nicola

Gabbi, Giampaolo

Gabriela, Dobrotă
Gaetano, Davide De

Gan, Christopher

Gan, Guojun

Garcia, Jennifer Alonso

García, Fernando

García-Álvarez De Perea, Juan

Garrido, María Del Carmen Calvo

Gasbarro, Domenic

Gennari, Francesca

Gete, Pedro

Gherghina, Ştefan Cristian

Ghosh, Subir

Ghulam, Yaseen

Gilli, Manfred

Glabadanidis, Paskalis

Glavee-Geo, Richard

Gogas, Periklis

Gomes, Orlando

Gómez, Georgina

Gradojevic, Nikola

Grima, Simon

Gruszczyński, Marek

$\mathrm{Gu}$, Alun

Guijarro, Francisco

Guillén, Montserrat

Guillotin, Bertrand

Gulasekaran, Rajaguru

Guo, Zach

GÜRCAN, Önder

Hadaś-Dyduch, Monika

Halicioglu, Ferda

Hall, Colin Michael

Hamori, Shigeyuki

Hanly, Jim

Harasheh, Murad

Harms, Philipp

Hassan, Rohail

Hassani, Hossein

Hategan, Camelia Daniela

Herzog, Bodo

Hkiri, Besma

Ho, Manh-Tung

Hong, Jin B.

Horák, Jakub

Horvatova, Eva

Hošková-Mayerová, Šárka

Huang, Cheng-Tsu

Huang, Chin-Huang

Iannuzzi, Antonia Patrizia

Indriawan, Ivan

Ishii, Hokuto

Ivascu, Larisa 
Jakovac, Pavle Janovec, Michal

Jiang, Yuanying

Jianu, Ionel

Jianu, Iulia

Johan, Sofia

Kaabia, Olfa

Karminsky, Alexander M.

Kenett, Dror

Khajeheian, Datis

Kim, Jong-Min

Kim, Jungmu

Kjærland, Frode

Klazar, Stanislav

Klein, Tony

Klimkiewicz, Katarzyna

Kocur-Bera, Katarzyna

Koehler, Matthew

Kolokolova, Olga

Kordoš, Marcel

Korol, Tomasz

Korzeb, Zbigniew

Kovacova, Maria

Kozhanov, Igor

Kutlu, Levent

Kwon, Roy H.

Kyriakou, Ioannis

Kyriazis, Nikolaos A.

Lagasio, Valentina

Lahmiri, Salim

Lando, Tommaso

Lars, Kirkby Justin

Laurila, Hannu

Leboeuf, Gael

Leccadito, Arturo

Lee, Jeong Hwan

Lee, Kuo-Jung

Lentner, Csaba

Li, Frank

Li, Kai

Li, Qi

Li, Jing

Li, Weiping

Li, Xiaofei

Li, Zhaohua

Li, Zhichuan (Frank)

Liang, Yi

Liczmańska-Kopcewicz, Katarzyna

Lim, Dongkuk

Lin, Ching Trong

Liu, Rui

Liu, Shuangzhe
Lizińska, Joanna

López-Gutiérrez, Carlos

Lu, Su-Lian

Lucarelli, Stefano

Lukason, Oliver

Lux, Nicole

Malhotra, D.K.

Man Li, Rita Yi

Mansor, Fadillah

Manta, Otilia

Marrouch, Walid

Marszk, Adam

Martínez, Francisco Guijarro

Marvão Pereira, Alfredo

Matthews, Kent

Matuszak, Łukasz

Matuzeviciute, Kristina

Mazzù, Sebastiano

McAleer, Michael

Men, Zhongxian

Méndez-Picazo, María-Teresa

Meszaros, John

Mets, Tõnis

Michalski, Grzegorz

Miller, Stephen Matteo

Minford, Patrick

Miras Rodríguez, María Del Mar

Molnar, Peter

Montllor, Joan

Moosa, Imad

Morea, Donato

Morgan, Peter J.

Morley, Bruce

Moutanabbir, Khouzeima

Mukhtarov, Shahriyar

Müller, Julian Marius

Muncharaz, Javier Oliver

Naldi, Maurizio

Newaz, Mohammad

Nguyen, Minh Hoang

Nicolò, Giuseppe

Nielsen, Jens Perch

Nigri, Giorgia

Novickytė, Lina

Nowak, Piotr

Obeid, Hassan

Ogawa, Eiji

Olkhov, Victor

Ortega, Jose Antonio

Pacana, Andrzej

Paccagnini, Alessia

Palečková, Iveta 
Paltrinieri, Andrea

Panagiotidis, Theodore

Panait, Mirela

Panetta, Ida Claudia

Panniello, Umberto

Pansera, Bruno A.

Park, Jaehwan

Partal Ureña, Antonio

Pastusiak, Radoslaw

Păunescu, Carmen

Pele, Daniel

PENG, Xuanhua

Perenyi, Aron

Pérez-Hernández, Francisco

Perote, Javier

Pesta, Michal

Pinho, Carlos

Pires Manso, Jose' Ramos

Plakandaras, Vasilios

Polanco-Martínez, Josué M.

Popescu, Gheorghe $\mathrm{H}$.

Porrini, Donatella

Port, Henry

Potrafke, Niklas

Pratt, Cornelius

Prusak, Błażej

Qiao, Zhuo

Quessy, Jean-François

Rakowski, David

Rambaccussing, Dooruj

Ramos, Célia M. Q.

Rea, William

Reimers, Hans-Eggert

Rjoub, Husam

Rodríguez Martín, José Antonio

Rój, Justyna

Rouatbi, Wael

Rusu, Valentina Diana

Saadaoui, Jamel

Sadorsky, Perry

Safa, M Faisal

Sahin, Afsin

Saizarbitoria, Iñaki Heras

Salampasis, Dimitrios

Salas, Jesus M.

Sanchez, Benito

Sanderson, Rohnn

Sawik, Bartosz

Scaillet, Olivier

Scaramozzino, Pasquale

Schoutens, Wim

Schramm, Marc
Sevic, Aleksandar

Shaikh, Imlak

Shen, Kao-Yi

Shen, Xuan (Shelly)

Shenai, Vijay

Shi, Xunpeng

Sim, Nicholas

Simões, Nádia

Singh, Madhusudan

Sinha, Amit

Skouloudis, Antonis

Škrinjarić, Tihana

Skvarciany, Viktorija

Ślusarczyk, Beata

Smaga, Pawel

Snudden, Stephen

SO, Jacky Yuk-Chow

Sonenshine, Ralph

Stafford, Frank

Stauvermann, Peter Josef

Stawska, Joanna

Steffensen, Mogens

Stentoft, Lars

Streimikiene, Dalia

$\mathrm{Su}$, Ender

Sultonov, Mirzosaid

Sun, Yiguo

Syriopoulos, Konstantinos

Szczepankiewicz, Elżbieta

Tajani, Francesco

Tan, Yong

Tang, Jian

Tarrant, Wayne

Tchatoka, Firmin Doko

Tomczak, Sebastian

Toyoshima, Yuki

Trapczynski, Piotr

Tselios, Vassilis

Tsinaslanidis, Prodromos

Tsionas, Mike G.

Tsolas, Ioannis E.

Tzeremes, Panayiotis

Urbaniec, Maria

Van Hove, Leo

Vezeris, Dimitrios

Vives, Josep

Vogiazas, Sofoklis

Voica, Marian Catalin

Vrbka, Jaromír

Vuong, Quan-Hoang

Wang, GangJin

Wang, Min 
Wang, Weining

Wang, Xiaoli

Weathers, Jamie

Weber, Christoph S.

Wegener, Christoph

Wen, Yuanji

Wieczorek-Kosmala, Monika

Wihlborg, Clas

Wohar, Mark E.

Wong, Wing-Keung

Wood, Pushpa

$\mathrm{Wu}, \mathrm{Kai}$

Wu, Kuop Jui

$\mathrm{Wu}$, Lifeng

$\mathrm{Wu}$, Xueping

Yang, Sheng
Yang, Weixin

Yang, Yipeng

Yao, Kai

Yao, Wenying

Yezer, Anthony

Yilmazkuday, Hakan

Yu, Billy T.W.

Yue, Qi

Zaremba, Adam

Zhang, Ren

Zhang, Yuejun

Zhao, Hongbiao

Zhao, Yichuan

Zhu, Fukang

Zubeltzu, Eugenio

(C) 2020 by the author. Licensee MDPI, Basel, Switzerland. This article is an open access article distributed under the terms and conditions of the Creative Commons Attribution (CC BY) license (http://creativecommons.org/licenses/by/4.0/). 